\title{
Method for Detection of the Lean Meat Ratio in Pork Carcass Based on Fiber Optic Sensor
}

\author{
Zhichao Sun, Sheng Leng, Wantai Ma, Qing Xu and Xiaoming Xie \\ College of Mechanical and Electrical Engineering, Nanjing University of Aeronautics and Astronautics, No. 29, Yudao Street, Nanjing 210016, \\ China
}

\begin{abstract}
One of the main evaluations in pork carcass is the lean meat ratio. A method based on Fiber optic sensor and NIR (Near Infrared) to acquire the ratio was put forward. This method acquired the reflected light intensity of the meat inside the pork carcass, by using double optical paths to reduce the errors caused by the instability of light. The fiber was lead into a probe which can be stabbed into the pork carcass, and then the reflected light was detected by a circuit named LIA (lock in amplifiers) to recognize the weak reflected light intensity effectively. The results suggested that the reflected light of the fat part of the pork was much higher than that of lean part, and through medium filter, the conditions of fluctuation was improved. The system was proved to be quick and accuracy for the detection of lean meat ration in pork carcass.
\end{abstract}

\section{Introduction}

Pork plays a very important role in the meat consumption in China. And with the development of society, people's requirements on the quality of pork meat becomes higher and higher. They prefer lean meat to fat meat, thus a pork's values mostly depends on the lean meat percentage. The percentage has become a very important indicator of a pork when grading the pork carcass [1]. To create a fair marketplace, many technologies had been applied into distinguishing fat meat and lean meat of pork such as ultrasonic technology, computer vision, opto-electronic, etc.

Based on Ultrasonic technology, SFK Company of Denmark developed UltraFom 300 to detect the reflected ultrasonic values of pork carcass [2]. Analyzing the different reflected values, the fat depth is got, and through collecting more than 3200 points of the pork carcass, a 3D ultrasonic image is established quickly. It can also get the estimation of marble score of pork carcass and the lean meat ratio automatically. This technology is widely used in European and American countries but seldom in China.

Computer vision is another technology used in pork carcass [3], [4]. Image-Meater is developed by CSB Company of German. It uses cameras to take photos of the pork carcass, analyzes the images and finds the relationship between image data and lean meat ratio. But the efficiency is not high as much as ultrasonic and opto-electronic.

Opto-electronic mainly uses the different reflected light values of fat and lean to detect the boundary and calculate the length of fat and lean meat. This technology is widely used in many companies like SFK, Hennessy and Destron Company.

The rapid development of the Near-infrared (NIR) Spectrum Detection technology provides a new method to detect the quality of meat nondestructively [5]. Organics or some inorganics are all made up of various molecules. In these molecules, there exists various groups such as $\mathrm{C}=\mathrm{C}, \quad \mathrm{N}=\mathrm{C}, \quad \mathrm{O}=\mathrm{C}, \quad \mathrm{O}=\mathrm{H}, \quad \mathrm{N}=\mathrm{H}$, and these groups' movements like expansion, vibration and bending all have their own frequency. Some power of the light will be absorbed by the groups. Most of the organic compounds of meat like protein, fat and moisture have different hydrogen groups. Therefore, the reflected light values are different for fat and lean meat.

As a new sensor, FOS [6] (fiber optic sensor) is widely used in measuring sound field, electric field, pressure, temperature and velocity. It has many talent characters such as high sensitivity, low transmission loss, etc. Therefore, this article improves the opto- electronic by applying fiber optic and probe into acquiring the intensity of the reflected light of the inside meat surface, distinguishing the fat and lean meat and acquiring the length of fat and lean meat finally.

\section{System design}

The main components of the meat is protein (including myofibril, myosin), water, fat, etc. These substances have different absorption or reflectance of light, thus the lean meat and fat meat under the same light should own different reflectance. According to the Kubelka-Munk theory, the reflectance can be defined as: 


$$
\ln \frac{1}{R}=\frac{S}{K}+B
$$

where $R$ represents the light reflectance of pork; $S$ and $K$ represent the absorption coefficient and scattering coefficients respectively; $\mathrm{B}$ is a constant. The reflectance $\mathrm{R}$ is defined as:

$$
\mathrm{R}=\frac{I}{I_{0}}
$$

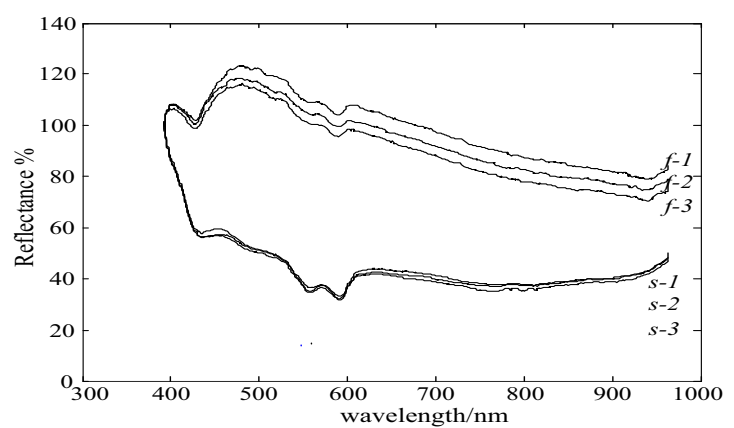

Figure 1. The different light reflectance of lean and fat meat

where $I$ represents the intensity of reflected light and $I_{0}$ represents the intensity of input light. Obviously the lean and fat meat have different $\mathrm{S}$ and $\mathrm{K}$ coefficients and Fig. 1 shows that $f-1, f-2, f-3$ are the reflectance of fat meat and $s-1, s-2, s-3$ are the reflectance of lean meat. Obviously the reflectance of fat are much higher than that of lean part which gives a method to detect the fat and lean meat.

\subsection{The design of FOS and Probe}

To avoid the errors caused by the light source, a double optical paths system based on fiber optic is designed to detect and measure the fat and lean meat. Different from measuring the light intensity directly, double optical paths method has two signal tunnels. One is called signal arm which transmit the light from light source to the inside of pork carcass through probe, and then to the light detector after being reflected, and the other is called reference arm which means the light goes to the light detector directly without being reflected. When the light power changes, two arms will help reduce the changes. The system is shown in Fig. 2.

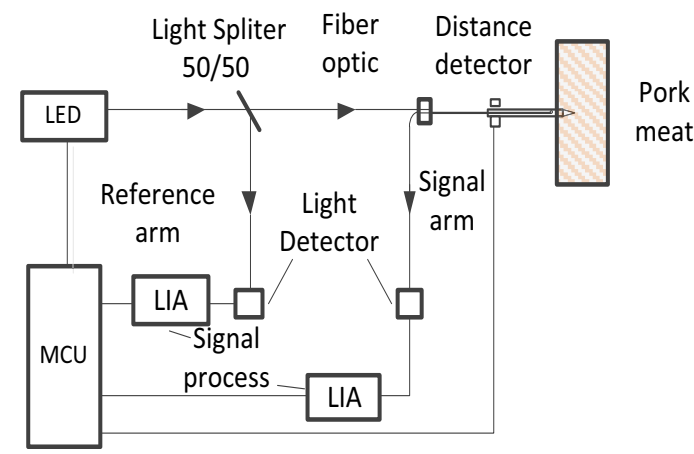

Figure 2. Double optical paths system

MCU controls the light source and receives the signals from light detectors and distance detector. The light splitter is to divide the light into two parts. One goes to the light detector directly, and the other goes to a probe which stabs into pork carcass to get reflected light from the surface of fat or lean meat and then goes back to another light detector. The reflectance then can be acquired by dividing signal arm values with reference arm values, and then the depth of probe into the pork carcass is measured by the distance detector.

The probe is designed and shown in Fig. 3. At the end of the probe, a blade is designed to stab into meat, and near the blade, there exists a small window for light. When the light arrives at the reflector glass through fiber optic, its direction changes and arrive at the surface of meat, and then get reflected and goes back to the fiber optic.

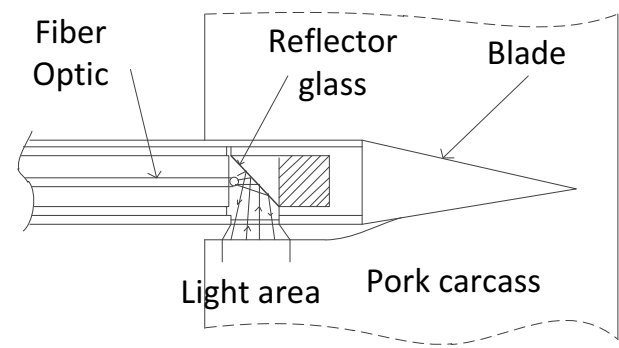

Figure 3. The design of puncture probe

\subsection{The detection of light signals}

The output power of the LED light is about $1.5 \mathrm{~mW}$ which is too weak to be detected, especially for the light which is absorbed. What's more, there are many noises in circuits caused by resistors, capacity and many other active devices. These noises are mixed with useful signals and when it is directly amplified, the noise will cover the light signals. Thus, a useful technology named Lock-in-amplifiers (LIA) is taken to help detect the weak signals [7]. The light signal is modulated by a high-frequency $(w)$ signal which can avoid $1 / f$ noise. When the whole signal is amplified, a BPF (ban-pass filter) filter out most of the low frequency noise and allow the useful signal pass. And then the PSD (phase-sensitive detector) help shift the frequency of modulated signal from $w$ to 0 . Then the $\mathrm{A} / \mathrm{D}$ converter will receive a $\mathrm{DC}$ signal after LPF (low-pass filter). The schematic diagram is shown in Fig. 4.

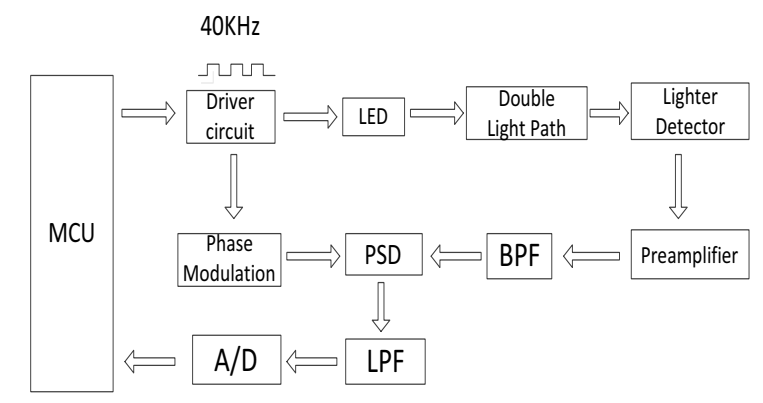

Figure 4. LIA detecting schematic

\subsection{Calculation of the length of fat and lean part}

When the probe fully pass through the carcass, a series of values are acquired. These values represents the different reflected light intensity. To judge the kinds of meat correctly, a threshold $\mathrm{V}$ is acquired. 


$$
\mathrm{V}=\frac{\sum \text { values }}{\text { Num }}
$$

It should be noticed that the values must be collected inside the pork carcass. Before calculating the length of the fat and lean part, the values in the air should be removed.

$$
f(v)= \begin{cases}v \geq V & \text { fat } \\ v<V & \text { lean }\end{cases}
$$

Eq. 2 shows how to distinguish the fat and lean part.

\section{Result and discussion}

Based on the theory above, a test experiment was constructed for checking the effectiveness and precision. The light source used LED (wavelength $=850 \mathrm{~nm}$ ), and the diameter of the fiber optic is $600 \mathrm{um}$. And the diameter of the probe is about $5 \mathrm{~mm}$. The meat samples must be fresh and have distinct boundary between the fat and the lean.

Taking different points of the pork carcass, the result is shown in Fig. 5.
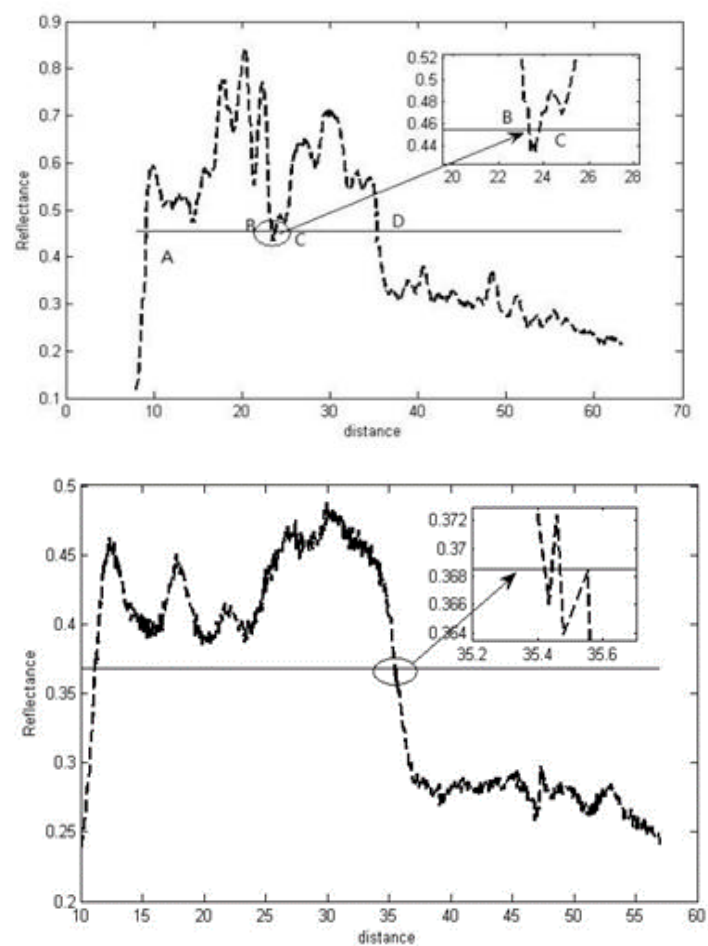

Figure 5. Result of the experiment (up: serious condition, down: ideal condition)

Apparently, the reflected light intensity of the lean is higher than that of the lean. However, the original data is difficult to calculate the lean meat ratio. The fluctuation of Fig. 5(up) is very serious, and there are some points which should be on the fat part but the values are under $\mathrm{V}$ line. The system will mistake this part as lean meat, then the length of the fat is L in Eq. 5.

$$
\begin{aligned}
\mathrm{L}=(\mathrm{B} . \text { distance }- \text { A. distance })+ \\
\text { (D. distance }- \text { C. distance })
\end{aligned}
$$

Fig. 5(down) shows the ideal conditions that the fat and lean are easily to tell apart. But at the boundary of fat and lean meat in Fig. 5(down), there exists a slight fluctuation which will increase the work to figure the lean meat ratio.

Obviously, these situations should be avoided as much as possible. To remove the influence of the serious fluctuation of the signals, a smooth filter algorithms-medium filter is chosen.

And the result is shown in Fig. 6. The solid line is the curve after medium filter and the dashed line is the original one. It can be seen that the local fluctuation is improved and the whole curve is smoother than before. Calculate all the cross points and acquire the length of all the fat and lean part like Eq. 5.
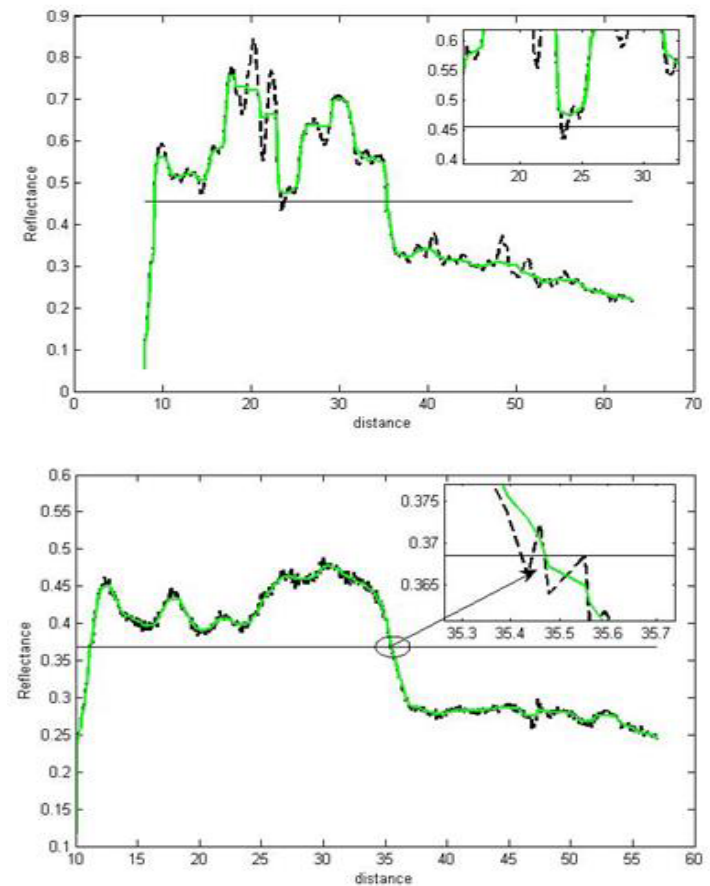

Figure 6. Result after medium filter (up: serious condition, down: ideal condition)

\section{Conclusion}

Through the experiment and discussion of the system designed, the length of the fat and lean part is detected, which proves the system to be effective. The NIR can not only be applied into the detecting the kinds of the meat, but also in the field of measuring the meat freshness, moisture content, etc. The average value V can help detect the kinds of pork meat correctly and the medium filter can be used in condition where the curves need to be smoothed effectively. By acquiring the fat and lean length of different parts of the pork carcass, the whole lean meat ratio of the pork then can be obtained. Through the integration of these methods, a kind of portable, low-cost and accurate solution can be applied in the food industry.

\section{Acknowledgement}

This work is being founded by the National Natural Science Foundation, China (No.51205201) and Innovation foundation for postgraduate students in 
Nanjing University of Aeronautics and Astronautics (No.ykfjj20150513).

\section{References}

1. N. Zhang, G. Zhou, X. Xu, Food Ferment. Ind., 31, $86(2005)$

2. J. Brodum, Can. J. Anim. Sci. 76, 443 (1998)

3. J. Yin, G. H. Zhou, X. L. Xu, Food Sci. (Beijing,
China), 31, 32(2010)

4. S. W. Zhao, Y. K. Peng, H. Y. Zhang, J. Food Saf. Qual, 3, 589(2012)

5. X. J. Yang, J. Y. Zhao, J. S. Zhao, Food Sci. Technol. (Beijing, China), 37, 267(2012)

6. F. Liu, W. He, F. Luo, L. Zhu, Semicond Optoelectron, 35, 928(2014)

7. Z. W. Tian, J. Q. Xiong, J. H. Pei, Control Instrum Chem. Ind, 5, 509(2014) 